\section{A residência de Medicina de Família e Comunidade no Brasil: Breve recorte histórico}

\author{
Family and Community Medicine residency in Brazil: Brief history \\ La residencia de Medicina de la Familia y Comunidad en Brasil: \\ Un breve histórico
}

\section{Resumo}

Introdução: A saúde no Brasil se estabeleceu como direito universal a partir da promulgação da Constituição da República Federativa do Brasil em 1988, o qual foi posteriormente ratificado e normatizado pela edição da Lei no 8080 de 1990, que dispõe sobre as condições para a promoção, proteção e recuperação da saúde, a organização e o funcionamento dos serviços correspondentes, conhecida por Lei Orgânica do Sistema Único de Saúde (SUS). Tendo como base os princípios norteadores foi lançado, pelo Ministério da Saúde, o Programa de Saúde da Família, em 1994, o qual se tornou Estratégia Saúde da Família, 12 anos após. No momento em que o país começou priorizar a Atenção Primária à Saúde (APS) como eixo central do sistema de saúde, assim como ocorre nos principais sistemas nacionais de saúde no mundo, percebeu-se a escassez de profissionais médicos formados para atuação nesse nível de atenção. Emergiu, então, a necessidade de ampliar o acesso à residência em Medicina de Família e Comunidade (MFC) no Brasil. Objetivo: Apresentar aspectos da constituição histórica dos Programas de Residência em MFC (PRMFC) brasileiros. Resultados: Nos primeiros anos do século XX, em diversas unidades federativas, houve considerável aumento de vagas de RMFC - com destaque para o PRMFC da Secretaria Municipal de Saúde do Rio de Janeiro -, abrangendo cidades que antes não contavam com essa formação. Tal contexto pode ser compreendido à luz da significativa articulação construída no âmbito do SUS, para a formação de recursos humanos em saúde. Conclusão: A Residência em MFC mantém-se em expansão no país e já pode ser encontrada em algumas das principais cidades do Brasil.

Palavras-chave: Atenção Primária à Saúde; Medicina de Família e Comunidade; Clínicos Gerais; Internato e Residência
Keith Bullia da Fonseca Simas ${ }^{a}$

Andréia Patrícia Gomes ${ }^{b}$

Patrícia Passos Simões ${ }^{c}$

Daniel Knupp Augusto ${ }^{d}$

Rodrigo Siqueira-Batista ${ }^{e}$

a Programa de Pós-graduação em Bioética, Ética Aplicada e Saúde Coletiva (PPGBIOS), Universidade Federal do Rio de Janeiro (UFRJ); Secretaria Municipal de Saúde do Rio de Janeiro. Rio de Janeiro, RJ, Brasil. keithsimas.smsrio@gmail.com (Autor correspondente)

b Departamento de Medicina e Enfermagem, Universidade Federal de Viçosa (UFV).

Viçosa, MG, Brasil.

andreiapgomes@gmail.com

- Secretaria Municipal de Saúde do Rio de Janeiro; Centro Universitário IBMR. Rio de Janeiro, RJ, Brasil. patriciasubge@gmail.com

' Sociedade Brasileira de Medicina de Família e Comunidade (SBMFC). Rio de Janeiro, RJ, Brasil.knupp.bh@gmail.com

e Departamento de Medicina e Enfermagem, Universidade Federal de Viçosa (UFV); Programa de Pós-graduação em Bioética, Ética Aplicada e Saúde Coletiva (PPGBIOS), Universidade Federal do Rio de Janeiro (UFRJ); Núcleo de Estudos em Ciências Médicas (NUMED), Curso de Medicina, Faculdade Dinâmica do Vale do Piranga (FADIP). Viçosa, MG, Brasil. Imecs@ufv.br

Fonte de financiamento:

Os autores são gratos ao CNPq, pelo apoio à pesquisa.

Parecer CEP:

não se aplica.

Conflito de interesses:

declaram não haver.

Procedência e revisão por pares:

revisado por pares.

Recebido em: 31/01/2018.

Aprovado em: 21/12/2018. 


\begin{abstract}
Introduction: Health in Brazil was established as a universal right from the promulgation of the Constitution of the Federative Republic of Brazil in 1988. Subsequently, ratified and standardized by the edition of the Law 8080 of 1990 , which provides the conditions for the promotion, protection and recovery of health, organization and functioning of the corresponding services, known as the organic law of the unique system of Health (SUS). Based on the guiding principles of SUS, the Family Health Program was launched by the Ministry of Health in 1994, which became the Family Health Strategy twelve years later. As the country began to prioritize Primary Health Care (PHC) as the central axis of the health system, as in the main national health systems in the world, the shortage of medical professionals trained to this level of care. The need to expand access to family and community medicine (MFC) in Brazil emerged. Objective: To present aspects of the historical constitution of the Residency Programs in Brazilian CFM (PRMFC). Results: In the first years of the $20^{\text {th }}$ century, in several federal units, there was a considerable increase in RMFC vacancies - with emphasis on the PRMFC of the Municipal Health Department of Rio de Janeiro -, covering cities that did not previously have this training. This context can be understood in the light of the significant articulation built within the SUS, for the formation of human resources in health. Conclusion: The MFC Residency continues to expand in the country and can already be found in some of the main Brazilian cities.
\end{abstract}

Keywords: Primary Health Care; Family Practice; General Practioners; Internship and Residency

\title{
Resumen
}

Introducción: La salud en Brasil se estableció como derecho universal a partir de la Constitución Federal de 1988, y fue posteriormente, ratificado y normalizado por la edición de la Ley no. 8080 de 1990, que dispone sobre las condiciones para la promoción, protección y la recuperación de la salud, la organización y el funcionamiento de los servicios correspondientes, conocida como Ley Orgánica del Sistema Único de Salud (SUS). Con base en los principios orientadores del SUS, fue lanzado por el Ministerio de Salud, el Programa de Salud de la Familia, en 1994, el cual se convirtió en Estrategia Salud de la Familia, doce años después. En el momento en que el país comenzó a priorizar la Atención Primaria de Salud (APS) como eje central del sistema de salud, así como ocurre en los principales sistemas nacionales de salud en el mundo, se percibió la escasez de profesionales médicos formados para actuación en ese nivel de salud atención. Se planteó la necesidad de ampliar el acceso a la residencia en Medicina de Familia y Comunidad (MFC) en Brasil. Objetivo: Presentar aspectos de la constitución histórica de los Programas de Residencia en MFC (PRMFC) brasileños. Resultados: En los primeros años del siglo XX, en diversas unidades federativas, hubo un considerable aumento de vacantes de RMFC - con destaque para el PRMFC de la Secretaría Municipal de Salud de Rio de Janeiro -, abarcando ciudades que antes no contaban con esa formación. Tal contexto puede ser comprendido por la significativa articulación construida en el ámbito del SUS, para la formación de recursos humanos en salud. Conclusión: La Residencia en MFC se mantiene en expansión en el país y ya puede ser encontrada en algunas de las principales ciudades de Brasil.

Palabras clave: Atención Primaria de Salud; Medicina Familiar y Comunitaria; Médicos Generales; Internado y Residencia

\section{Introdução}

A Conferência Internacional sobre Atenção Primária à Saúde (APS) em Alma Ata (1978), organizada pela Organização Mundial da Saúde (OMS), estabeleceu o conceito de 'saúde para todos', convocando a totalidade dos países a revisar os respectivos sistemas de saúde, tornando-os acessíveis à população. ${ }^{1}$ Um longo caminho foi percorrido até a Carta de Ottawa, em 1986, e a Declaração de Adelaide, em 1988 elaboradas por ocasião da primeira e da segunda Conferências Internacionais sobre Promoção da Saúde -, as quais apresentam uma construção coletiva sobre os conceitos fundamentais abordados no contexto da Promoção da Saúde, realizado em várias partes do mundo. ${ }^{1}$

No Brasil, a saúde demorou a se configurar como um direito universal, o que ocorreu com a promulgação da Constituição Federal (CF) de 1988, a qual passou a considerar a saúde como "um direito de todos e um dever do Estado". Na Carta Magna, adota-se, também, o princípio da igualdade, de acordo com o qual "todos são iguais perante a lei, sem distinção de qualquer natureza" (art. $5^{\circ}$ ). Também estabelece, em seu artigo $3^{\circ}$, que constituem objetivos fundamentais do estado, "erradicar a pobreza e a marginalização e reduzir as desigualdades sociais e regionais", bem como "promover o bem de todos sem preconceitos de origem, raça, sexo, cor, idade e quaisquer outras formas de discriminação". ${ }^{2}$ Dois anos depois, a Lei Orgânica do Sistema Único de Saúde (SUS) (Lei 8.080/1990) entra em vigor para disciplinar o mandamento constitucional. ${ }^{3}$ 
Importante ressaltar que a igualdade é um princípio essencial ao pleno exercício da cidadania, ao se reconhecer que todos os cidadãos são detentores dos mesmos direitos. Essa perspectiva deve ser complexificada, no bojo dos debates acerca da justiça, em termos da equidade, conceito atrelado à ideia de justiça social e que permite reconhecer que os indivíduos são diferentes entre si, necessitando - por exemplo - de cuidado diferenciado, de modo a eliminar ou reduzir as desigualdades existentes.

Ao revés, desigualdade e a iniquidade devem ser compreendidas na perspectiva da injustiça, domínio no qual há desrepeito aos direitos fundamentais e a dignidade da pessoa. Assim, reduzir desigualdades - frente à impossibilidade de eliminá-las - deve ser o objetivo central de toda política pública, que deverá transformar a igualdade formal da lei em igualdade material de fato. ${ }^{4}$

Em conformidade com os princípios norteadores do SUS, especialmente a equidade, o Brasil lança, em 1994, o Programa Saúde da Família (PSF), embora se tenha notícia de que já nesta data, guardadas suas particularidades, haviam sido implantados programas similares em alguns municípios, entre eles, Niterói (RJ), em 1991, e Itacarambi (MG), em 1993. ${ }^{5}$

O PSF tinha por objetivo a reorientação do modelo de atenção à saúde vigente, com ampliação do acesso às demandas de saúde, integralidade das ações de saúde e longitudinalidade da atenção, organizando o sistema e as redes de cuidado a partir da Atenção Primária à Saúde (APS), ${ }^{6}$ e fazendo com que a saúde fosse reconhecida como um direito de cidadania e, portanto, expressão da qualidade de vida. ${ }^{7}$ Do mesmo modo, a APS diz respeito ao comprometimento dos governos em atender às necessidades em saúde da população por meio de serviços de altas qualidade e resolutividade. ${ }^{8}$

As propostas de reformulação da APS no Brasil - por parte do Governo Federal, dos estados e dos municípios - previa a instalação de 40 mil equipes de Saúde da Família. Estas equipes deveriam estar formadas por diversos profissionais: médico(a), enfermeiro(a), técnicos(as) de enfermagem e agentes comunitários de saúde (ACS), os quais desenvolveriam o trabalho de forma multi e interdisciplinar a fim de prover cuidados aos indivíduos e à comunidade de uma área adscrita, o território. Nesse contexto, o profissional médico passa a ser considerado um recurso crítico, uma vez que sua formação pode impactar fortemente no desejo de vincular-se a tal tarefa - o trabalho na APS -, assim como ser inadequada ou insuficiente para que possa exercê-la com sucesso e efetividade..$^{9,10}$

Os debates sobre a educação do profissional da medicina com aptidão para atuar na APS/ Estratégia Saúde da Família (ESF) se dirigem para a necessidade da formação de um médico geral ou generalista General Practitioner (GP) -, cuja origem pode ser recuperada a partir da realidade inglesa. De fato, após a Segunda Guerra Mundial, tal trabalhador tornou-se a pedra angular do National Health Service (NHS), o sistema nacional de saúde da Inglaterra, surgido em 1948. Os GP começaram a trabalhar junto às autoridades distritais e aos órgãos de controle dos serviços de APS, ${ }^{11}$ em um contexto no qual o NHS provia cobertura universal, baseada nos princípios de equidade e de integralidade. Os médicos da APS recebiam por capitação, isto é, conforme o número de pacientes que compunham sua lista. Os GP proviam serviços ambulatoriais e agiam como porta de entrada (gatekeepers) para assistência hospitalar não emergencial. Os pacientes podiam estar ligados a apenas um GP. ${ }^{12} \mathrm{O}$ termo general practitioner de hoje é o que fez residência médica, que corresponderia, no Brasil, à Medicina de Família e Comunidade (MFC). ${ }^{13}$

A figura do médico de família talvez simbolize ainda hoje uma imagem idealizada da medicina. Esse tipo de prática se configurou com formas e importância distintas em diversos países. ${ }^{11}$ No início dos anos 
50 do século $X X$, suas características básicas estavam relacionadas à ideia de que se deveriam alcançar os pacientes precocemente no ambiente domiciliar, provendo cuidados contínuos, incluindo a prevenção das doenças, fossem elas de natureza mental ou física. ${ }^{10} \mathrm{Em} \mathrm{1966}$, quatro relatórios lançaram as bases para a criação da nova especialidade. ${ }^{10}$

A MFC prima pela integralidade, com foco na APS, o que a torna um componente primordial desse nível de atenção do SUS. ${ }^{14-16}$ Por isso, é uma especialidade estratégica na conformação dos sistemas de saúde. ${ }^{14,15,17}$ Cabe à MFC - uma vez que atua nos cuidados primários, em geral, o primeiro acesso ao sistema de saúde -, cuidar de forma longitudinal, integral e coordenada da saúde das pessoas, considerando seu contexto familiar e comunitário.

Os princípios e as práticas da MFC são centrados na pessoa, na relação entre médico e indivíduo, e na relação deste sujeito, mais ou menos sadio, com sua família e com a comunidade. ${ }^{18} \mathrm{O}$ canadense McWhinney, considerado um dos maiores estudiosos da MFC, definiu quatro competências próprias dessa especialidade: (1) solução de problemas não diferenciados; (2) competências preventivas; (3) competências terapêuticas (de problemas frequentes de saúde); (4) competência de gestão de recursos. ${ }^{16}$

Com base nessas considerações, o objetivo do presente artigo é apresentar um breve histórico sobre os programas de residência em MFC, enfocando - especialmente - o Programa de Residência em MFC da Secretaria Municipal de Saúde do Rio de Janeiro (SMS-RJ), uma vez que esta é, atualmente, a cidade brasileira com o maior número de vagas de residência em Medicina de Família e Comunidade. ${ }^{19}$

\section{Residência de MFC no Brasil: as origens}

As origens da Medicina de Família e Comunidade no Brasil podem ser divididas, segundo Falk, ${ }^{20}$ em cinco fases:

1. 1974-1980 - nesta primeira etapa, a especialidade - e a residência médica - não tinham ainda nome definido e nenhum respaldo normativo ou legal. Existiam poucos Programas de Residência Médica (PRM) no país, incluindo os três pioneiros, iniciados em 1976: (i) Programa de Residência em Multiprofissional em Saúde Comunitária (hoje: Residência Integrada em Saúde), Centro de Saúde Escola Murialdo (Porto Alegre - RS); (ii) Programa de Residência em Medicina Geral e Comunitária. Projeto Vitória (Vitória de Santo Antão, próximo a Recife - PE), extinto em 1986; e (iii) Programa de Residência em Medicina Geral e Comunitária, Serviço de Medicina Integral da UERJ (Rio de Janeiro - RJ).

2. 1981 a 1985 - essa fase inicia com a Resolução 07/81 da Comissão Nacional de Residência Médica (CNRM), em 1981, que formaliza os PRM em Medicina Geral Comunitária (MGC), oficializando esta área como uma especialidade no Brasil, com esta denominação.

3. 1986 a 1994 - durante a $8^{\text {a }}$ Conferência Nacional de Saúde, em março de 1986, em Brasília, é reativada a Sociedade Brasileira de Medicina Geral Comunitária (SBMGC), sendo eleita nova Diretoria, com médicos gerais comunitários, passando a sede para Porto Alegre, RS.

4. 1995 a 2000 - nesta etapa, a partir do advento do Programa Saúde da Família (PSF) e de outras iniciativas, as áreas voltadas à APS se expandem muito, em uma velocidade bem maior que a 
capacidade de formação de pessoal. Em outubro de 2000, com o apoio de entidades de Portugal, ocorre o "Primeiro Encontro Luso-Brasileiro de Medicina Geral, Familiar e Comunitária" no Rio de Janeiro, RJ. Na assembleia final foi aprovada a proposta de reaglutinação da categoria, através do fortalecimento da SBMGC. Também ficou decidido que deveria ser intensificado o debate já iniciado sobre a conveniência da mudança de nome da especialidade.

5. 2001 a 2004 - essa fase se inicia com a terceira reativação da Sociedade Brasileira de Medicina de Família e Comunidade (SBMFC), em março de 2001, destacando-se que nova diretoria é eleita, com membros do RS, RJ e PR e sede em Porto Alegre.

A SBMFC foi fundada em dezembro de 1981, com Estatuto registrado em Cartório na cidade de Petrópolis, RJ, sob o antigo nome de MEDICINA GERAL COMUNITÁRIA. Tornou-se representante da especialidade médica junto ao Conselho Federal de Medicina (Diário Oficial de 23 de outubro de 1986) e, mais recentemente, em novembro de 2003, junto à Associação Médica Brasileira - AMB. ${ }^{16}$

Atualmente, pode-se identificar uma sexta fase [acrescida, pelos autores do presente artigo, à original descrição de Falk] ${ }^{20}$ - 2004 até os dias atuais. Nesta fase, pode-se destacar, em 2005, a Portaria 1.143 do Ministério da Saúde, que vem apoiar programas de residência médica em MFC, por meio de bolsas para a Educação pelo Trabalho; ${ }^{21}$ o I Congresso Paulista de Medicina de Família e Comunidade, o qual possibilitou um espaço de discussão e de compartilhamento de narrativas entre as Instituições de Ensino Superior com interesse e práticas na atividade. ${ }^{18}$

Destacam-se também iniciativas para a formação de especialistas: curso de especialização em Medicina de Família da própria Sociedade Brasileira de Medicina de Família (SOBRAMFA), criado em 2003;22 a Oficina de Formação de Preceptores em MFC, da SBMFC, iniciada em $2005^{23}$ e que já formou mais de mil preceptores ao longo dos últimos anos, os cursos de especialização em MFC de diversas instituições, como a Fundação de Ensino e Pesquisa em Ciências da Saúde, Instituto Brasileiro de Saúde da Família, Pontifícia Universidade Católica do Paraná, Secretaria de Estado da Saúde do Tocantins, Universidade de Brasília, Universidade Estadual Paulista, Universidade Federal do Paraná e Universidade Federal de Pernambuco, dentre outras, que ministram cursos de especialização em todas as regiões do país. ${ }^{6}$

Ainda nessa sexta fase, em 2007, ressalta-se o $6^{\circ}$ Concurso para Título de Especialista em Medicina de Família e Comunidade, em junho de 2007, com prova policêntrica aplicada, simultaneamente, em dez cidades do país, e o $7^{\circ}$ Concurso para Título de Especialista em Medicina de Família e Comunidade, em dezembro de 2007, com prova policêntrica aplicada, simultaneamente, em 32 cidades do país. Um marco de destaque foi em 2008, o $9^{\circ}$ Congresso Brasileiro de Medicina de Família e Comunidade, em Fortaleza, Ceará, e a prova do $8^{\circ}$ Concurso (agora denominado "Exame de Suficiência”) para Título de Especialista em Medicina de Família e Comunidade. ${ }^{24}$

A consolidação da SBMFC foi essencial nesse processo. De fato, a Sociedade é a entidade nacional que congrega os médicos que atuam em unidades e outros serviços de APS - incluindo aqueles da ESF -, prestando atendimento médico geral, integral e de qualidade aos indivíduos, às famílias e às comunidades. ${ }^{25}$ Incluem também professores, preceptores, pesquisadores e outros profissionais que atuam ou estão interessados nesta área. A SBMFC trabalha pelo fortalecimento do movimento dos profissionais que atuam na APS. ${ }^{22}$ 
A SBMFC é filiada às principais sociedades e organizações da especialidade em nível mundial, a saber: (a) Confederação Ibero-Americana de Medicina Familiar (CIMF); (b) Organização Mundial dos Médicos Gerais, de Família e Comunidade (WONCA); (c) Associação Saúde em Português (sede em Portugal); (d) "Action in International Medicine", com sede na Inglaterra. ${ }^{16}$

\section{Programas de residência de MFC credenciados no Brasil}

A residência médica é uma modalidade de ensino de pós-graduação destinada a médicos, instituída pelo Decreto $\mathrm{n}^{\circ} 80.281$, de 5 de setembro de 1977. Funciona em instituições de saúde, sob a orientação de profissionais médicos de elevada qualificação ética e profissional. O mesmo decreto criou a Comissão Nacional de Residência Médica (CNRM). O Programa de Residência Médica confere, ao médico residente, o título de especialista. A expressão "residência médica" só pode ser empregada para programas que sejam credenciados pela Comissão Nacional de Residência Médica. ${ }^{26}$

Baseada em princípios, conceitos e recomendações internacionais formalizadas pela Organização Mundial dos Médicos de Família - WONCA - a especialidade Medicina de Família e Comunidade tem por objetivos: 16

“

i. atuar, prioritariamente, no âmbito da APS, a partir de uma abordagem biopsicossocial do processo saúde-adoecimento;

ii. desenvolver ações integradas de promoção, proteção, recuperação da saúde no nível individual e coletivo;

iii. priorizar a prática médica centrada na pessoa, na relação médico-paciente, com foco na família e orientada para comunidade, privilegiando o primeiro contato, o vínculo, a continuidade e a integralidade do cuidado na atenção à saúde;

iv. coordenar os cuidados de saúde prestados a determinado indivíduo, família e comunidade, referenciando, sempre que necessário, para outros especialistas ou outros níveis e setores do sistema, mas sem perda do vínculo;

v. atender, com elevado grau de qualidade e resolutividade, no âmbito da Atenção Primária à Saúde, cerca de $85 \%$ dos problemas de saúde relativos a uma população específica, sem diferenciação de sexo ou faixa etária;

vi. desenvolver, planejar, executar e avaliar, junto à equipe de saúde, programas integrais de atenção, objetivando dar respostas adequadas às necessidades de saúde de uma população adscrita, tendo por base metodologias apropriadas de investigação, com ênfase na utilização do método epidemiológico;

vii. estimular a resiliência, a participação e a autonomia dos indivíduos, das famílias e da comunidade; viii. desenvolver novas tecnologias em atenção primária à saúde;

ix. desenvolver habilidades no campo da metodologia pedagógica e a capacidade de auto aprendizagem;

x. desenvolver a capacidade de atuação médica, relevando seus aspectos científicos, éticos e sociais." 16 
A Lei 12.871, de 2013, que instituiu o Programa Mais Médicos para o Brasil (PMMB), veio para ampliar a formação de especialistas em MFC, adequar essa mão de obra às necessidades da população e promover a universalização do acesso à residência médica. A Lei determina que, até o final de 2018, haja o mesmo número de vagas de residência de acesso direto que o número de egressos dos cursos de graduação do ano anterior. ${ }^{27}$ A aprovação das novas Diretrizes Curriculares Nacionais do Curso de Graduação em Medicina, em 2014, ${ }^{28}$ assim como a ampliação de vagas de graduação e de residência compõem, igualmente, esse novo cenário instituído pela referida lei.

Podem-se enumerar algumas vantagens que torna a Residência de Medicina de Família e Comunidade (RMFC) atrativa aos profissionais médicos: o residente que ingressou, a partir de 2015, na RMFC, e concluiu os dois anos do programa passou a ter direito a pontuação adicional de $10 \%$ nos processos seletivos de residência para outras especialidades, a partir de março de 2016;29 o residente que ingressar na RMFC e se inscrever em um curso de formação de preceptores, parte do Plano Nacional de Formação de Preceptores, com duração de dois anos receberá, enquanto permanecer no curso, uma bolsa mensal. Muitos PRMFC têm formatos nos quais os proponentes do programa complementam a bolsa, garantindo uma remuneração que, no total, chega ao dobro ou mesmo ao triplo do valor da bolsa de residência no Brasil. Ademais, houve um importante aumento de vagas de RMFC, com vários programas em novas cidades que antes não contavam com essa formação. Assim, torna-se possível realizar a residência mais próxima do seu local de atuação. ${ }^{30}$

A Tabela 1 apresenta os estados/municípios que possuem o PRMFC credenciados, no Brasil. Serão comentados, brevemente, programas vinculados a secretarias de saúde do país, na perspectiva de que os mesmos exprimem uma significativa articulação com o SUS para a formação de recursos humanos em saúde, com destaque para o PRMFC da Secretaria Municipal de Saúde do Rio de Janeiro, o qual - atualmente - conta com o maior número de vagas e se insere em uma inovadora modalidade de se pensar/agir em termos de APS/ESF no Brasil: as Clínicas de Família. ${ }^{31}$

O Gráfico 1 e a Tabela 2 demonstram a evolução histórica dos PRMFC credenciados, no Brasil, entre os anos de 2002 a 2016. É possível perceber que o total de vagas por ano apresenta uma elevação ao longo do tempo, passando de 193 vagas em 2002 para 2.785 no ano de 2016. Destacam-se, nesses anos, a associação entre períodos de maior crescimento de vagas com políticas de incentivo à formação de MFC, como no intervalo de 2005 para 2006, por influência do PET-Saúde, no intervalo de 2011 para 2012, por influência do Pró-Residência, e a partir de 2014, por influência do PMMB. Merece ainda destaque, em particular, o aumento de vagas ocorrido de 2015 para 2016, que foi fruto de uma intensa atividade de estímulo à ampliação de vagas e credenciamento de novos programas, realizada por meio de uma colaboração do Ministério da Educação com a SBMFC e suas associações estaduais.

\section{PRMFC da Secretaria Municipal de Saúde (SMS) de Curitiba}

A cidade de Curitiba começou a organizar a APS já na década de 1980. Pela implantação de unidades de saúde, um médico generalista figurava ao lado de outros profissionais, atendendo a uma população específica. ${ }^{32}$ 
Tabela 1. Distribuição dos Programas de Residência de Medicina de Família e Comunidade credenciados no Brasil, segundo Estados e Municípios.

\begin{tabular}{|c|c|}
\hline Unidades/Estados & Municípios \\
\hline Acre & Rio Branco \\
\hline Alagoas & Maceió \\
\hline Bahia & $\begin{array}{l}\text { Feira de Santana, Itabuna, Luis Eduardo Magalhães, Mucuri, Porto Seguro, Salvador, Teixeira de Freitas, } \\
\text { Vitória da Conquista }\end{array}$ \\
\hline Espirito Santo & Colatina, Vitória, Vila Velha \\
\hline Goiás & Anápolis, Goiânia \\
\hline Maranhão & Caxias, São Luis \\
\hline Minas Gerais & $\begin{array}{l}\text { Alfenas, Araguari, Belo Horizonte, Betim, Bom Despacho, Brumadinho, Capelinha, Caratinga, Contagem, } \\
\text { Divinópolis, Governador Valadares, Itajubá, Juiz de Fora, Lagoa Santa, Montes Claros, Muriaé, Ouro Preto, } \\
\text { Paracatu, Poços de Caldas, Pouso Alegre, Santo Antônio do Amparo, Santo Antônio do Monte, São João Del } \\
\text { Rei, Sete Lagoas, Uberaba, Uberlândia, Vespasiano, Viçosa }\end{array}$ \\
\hline Mato Grosso & Cuiabá, Várzea Grande, Sinop, Lucas do Rio Verde \\
\hline Pará & Belém, Santarém \\
\hline Paraíba & Campina Grande, Cajazeiras, João Pessoa, Patos \\
\hline Pernambuco & Recife, Petrolina, Caruaru, Jaboatão dos Guararapes \\
\hline Paraná & $\begin{array}{l}\text { Apucarana, Campo Largo, Campo Mourão, Cascavel, Curitiba, Foz do Iguaçu, Guarapuava, Londrina, } \\
\text { Maringá, Pato Branco, Ponta Grossa, São José dos Pinhais, Sarandi, Umuarama }\end{array}$ \\
\hline Rio de Janeiro & $\begin{array}{l}\text { Angra dos Reis, Barra Mansa, Campos dos Goytacazes, Duque de Caxias, Macaé, Niterói, Petrópolis, Rio de } \\
\text { Janeiro, Teresópolis, Três Rios, Valença, Vassouras, Volta Redonda }\end{array}$ \\
\hline Rio Grande do Norte & Natal, Mossoró, Santa Cruz \\
\hline São Paulo & $\begin{array}{l}\text { Araraquara, Araras, Botucatu, Campinas, Catanduva, Fernandópolis, Franca, Guarulhos, Indaiatuba, Ipuã, } \\
\text { Jacareí, Jundiaí, Limeira, Marília, Mauá, Mogi das Cruzes, Olímpia, Piracicaba, Praia Grande, Ribeirão Preto, } \\
\text { Rio Claro, Santo André, São Bernardo do Campo, São Carlos, São José do Rio Preto, São José dos Campos, } \\
\text { São Paulo, Sorocaba, Taubaté, Valinhos, Votuporanga }\end{array}$ \\
\hline Tocantis & Araguaína, Gurupi, Palmas, Porto Nacional \\
\hline
\end{tabular}

Fonte: Comissão Nacional de Residência Médica. Brasil, 2016.

A despeito de Curitiba ter uma história de mais de 30 anos do médico generalista na APS e de contar com uma residência em MFC nas suas unidades de saúde (a da PUC-PR), percebeu-se a necessidade de ampliar o número de vagas e de responsabilizar diretamente a gestão municipal por essa tarefa. Com efeito, em janeiro de 2013, foi criada a Comissão de Residência Médica (COREME) da SMS de Curitiba e, em abril, os 12 primeiros residentes de MFC iniciaram as atividades. Uma das características desse PRM tem sido a de oferecer essa possibilidade para os médicos concursados da rede.

Desde essa implementação, já se percebe - como efeito imediato - uma melhoria na qualidade dos serviços nas unidades de saúde que contam com a residência. ${ }^{32}$ 


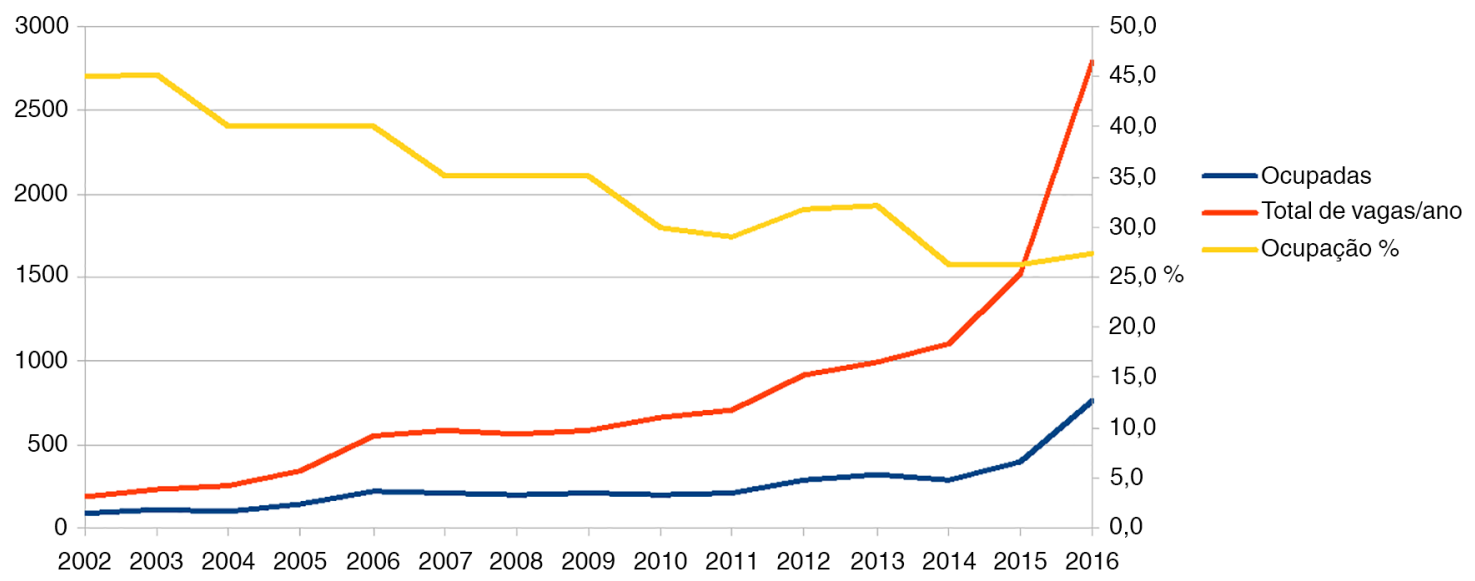

Gráfico 1. Evolução histórica dos Programas de Residência de Medicina de Família e Comunidade credenciados no Brasil - 2002 a 2016. Fonte: Comissão Nacional de Residência Médica. Brasil, 2016.

Tabela 2. Evolução histórica dos Programas de Residência de Medicina de Família e Comunidade credenciados no Brasil - 2002 a 2016.

\begin{tabular}{lcccccccc}
\hline Ano & $\mathbf{2 0 0 2}$ & $\mathbf{2 0 0 3}$ & $\mathbf{2 0 0 4}$ & $\mathbf{2 0 0 5}$ & $\mathbf{2 0 0 6}$ & $\mathbf{2 0 0 7}$ & $\mathbf{2 0 0 8}$ & $\mathbf{2 0 0 9}$ \\
\hline Ocupadas & 87 & 105 & 100 & 138 & 219 & 205 & 196 & 205 \\
Total de vagas/ano & 193 & 232 & 249 & 345 & 547 & 585 & 558 & 584 \\
Ocupação \% & 45,1 & 45,3 & 40,2 & 40 & 40 & 35 & 35,1 & 35,1 \\
Ano & 2010 & 2011 & 2012 & 2013 & 2014 & 2015 & 2016 & Total \\
Ocupadas & 198 & 206 & 290 & 320 & 290 & 400 & 763 & 3.722 \\
Total de vagas/ano & 660 & 711 & 911 & 997 & 1100 & 1520 & 2785 & 11.977 \\
Ocupação \% & 30 & 29 & 31,8 & 32,1 & 26,4 & 26,3 & 27,4 & - \\
\hline
\end{tabular}

Fonte: Comissão Nacional de Residência Médica. Brasil, 2016.

\section{PRMFC da Secretaria Municipal de Saúde de Florianópolis}

A cidade de Florianópolis - historicamente - tem se destacado pelo investimento na APS, estando sempre entre as capitais com maior cobertura em Saúde da Família (SF) do Brasil. Além disso, o município vem investindo na qualificação das equipes já existentes através de um processo de educação continuada para seus servidores e do fomento à formação profissional, com a integração ensino-serviço em parceria com a Universidade Federal de Santa Catarina, na graduação. ${ }^{33}$

Outra iniciativa que merece destaque foi a criação de uma residência própria, em MFC, com 16 vagas anuais. Como fator preponderante para a manutenção da qualidade das equipes de Saúde da Família, o município há 10 anos decidiu pagar diferenciadamente quem é especialista em MFC e tem realizado rotineiramente concursos públicos com este pré-requisito para preencher as vagas que surgem na rede de APS. Trata-se, portanto, de uma forma de atestar que a atuação, efetiva, na formação desses profissionais, é uma necessidade, uma decisão estratégica para qualquer município de médio e grande porte que queira contar com uma APS qualificada. Desse modo, Florianópolis tem uma característica diferenciada da maioria dos municípios do país, pois conta com grande parte das suas equipes compostas 
por médicos especialistas em MFC. Sendo assim, ela não sofre o mesmo problema que outros municípios enfrentam em encontrar preceptores para a implementação do programa. O maior desafio foi obter espaço nas unidades para a atuação dos médicos residentes. ${ }^{33}$

\section{PRMFC da Secretaria Municipal de Saúde de Fortaleza}

O PRMFC da Secretaria Municipal de Saúde de Fortaleza teve início em março de 2006. O Programa objetiva, principalmente, a formação de médicos com as competências necessárias para atuar na especialidade de Medicina de Família e Comunidade na rede municipal de saúde de Fortaleza, compondo as equipes de Saúde da Família e, consequentemente, aumentando a resolubilidade dos serviços de APS, possibilitando, ao mesmo tempo, a criação de espaços de práticas concretos e qualificados de formação de pessoal. ${ }^{34}$

O programa foi criado com uma proposta de duração de dois anos, prevendo atividades teóricas e práticas realizadas em serviço (unidades básicas de saúde) e na comunidade (visita domiciliar). As atividades de treinamento ocorrem em um contexto complexo e multidisciplinar de interações, envolvendo educadores, residentes, profissionais de áreas diversas do conhecimento (enfermeiros, odontólogos, assistentes sociais, auxiliares de enfermagem, agentes comunitários de saúde), usuários, comunidade e instituições. ${ }^{34}$

Todas as atividades práticas são supervisionadas diretamente por preceptores, os quais são médicos especialistas - pediatras, ginecologistas, clínicos gerais, psiquiatras e médicos de família e comunidade da rede de atenção à saúde da SMS. As aulas teóricas são ministradas por professores das universidades parceiras. A concepção de formar profissionais com uma visão de vigilância à saúde e que prestem uma atenção integral à população, com um olhar no indivíduo e em seu contexto social, já se encontra contemplada nos projetos político-pedagógicos dos cursos de graduação na área da saúde, porém precisa ser efetivada em ações como as propostas pelo programa do curso de RMFC. ${ }^{34}$

\section{PRMFC da Secretaria Municipal de Saúde do Rio de Janeiro}

O município do Rio de Janeiro contava, em 2010, com três tradicionais programas de residência médica em MFC: UERJ (10 vagas anuais), UFRJ (quatro vagas anuais) e ENSP (duas vagas anuais), com grande qualidade na formação. O programa da UERJ é um dos mais antigos do país, se não o mais antigo. ${ }^{35}$

A Subsecretaria de Promoção, Atenção Primária e Vigilância em Saúde da SMS Rio de Janeiro investiu em parcerias com cursos de graduação e aumentou o número de vagas para residência em MFC. Foi então criado um programa da Secretaria Municipal de Saúde para dar efetividade ao planejamento, ${ }^{19,36}$ amplificar a qualificação do médico que trabalha nas equipes de saúde da família cariocas e ampliar a oferta desse especialista para atuar na APS do município. ${ }^{34}$ A Tabela 3 apresenta uma série histórica da oferta de vagas pelo programa de Residência em Medicina de Família e Comunidade da SMS-RJ, instituído a partir de 2012.

Ao longo de dois anos, o PRMFC-RJ permitiu a formação e qualificação de profissionais, muitos dos quais se mantêm vinculados à realidade carioca, contribuindo para a qualificação e para a expansão da APS no município do RJ. Tal fato pode ser comprovado com a incorporação, à APS, de 27 médicos provenientes da primeira turma do Programa de Residência de Família e Comunidade do Município do Rio de Janeiro, seis deles como preceptores. ${ }^{36}$ 
Tabela 3. Série histórica de oferta de vagas e lotação do Programa de Residência de Medicina de Família e Comunidade da SMS-RIO.

\begin{tabular}{ccccc}
\hline \multirow{2}{*}{ Ano } & $\mathbf{N}^{\circ}$ de vagas & $\mathbf{N}^{\circ}$ de Inscritos & $\mathbf{N}^{\circ}$ de vagas \\
\cline { 2 - 5 } & Ofertadas em Edital & para a Seleção & Preenchidas \\
\hline \multirow{3}{*}{ PRMFC SMS-RIO } & $2012\left(^{*}\right)$ & 60 & 155 & 51 \\
& 2013 & 60 & 157 & 51 \\
& 2014 & 100 & 163 & 64 \\
& 2015 & 100 & 412 & 100 \\
& 2016 & 150 & 426 & 131 \\
\hline
\end{tabular}

$\left.{ }^{\star}{ }^{\star}\right)$ Ano de credenciamento do PRMFC da SMS-RIO junto a CNRM. Fonte: Parecer CNRM № 100/2011, Parecer CNRM № 1469/2014, Parecer CNRM № 169/2016 e Relatórios da Gerência de Desenvolvimento Técnico Acadêmico da SMS-RIO.

Diante do crescimento do programa - e atendendo de forma reativa à demanda de formação de especialistas na área -, as universidades e os serviços ligados às secretarias de saúde já conseguiram credenciar, junto à Comissão Nacional de Residência Médica, quinhentas bolsas de especialização nesta modalidade, incluindo o primeiro e o segundo ano de residência. ${ }^{10}$

\section{Conclusão}

O presente artigo buscou expor uma apreciação sucinta dos Programas de Residências de Medicina de Família e Comunidade no Brasil, no atual contexto das políticas públicas de saúde do país, com ênfase na APS, em especial à medicina de família e comunidade, tendo como protagonista a Estratégia Saúde da Família. Este modelo de APS adotado no Brasil, em franca expansão no município do Rio de Janeiro, exige uma ampliação no número de profissionais com formação em MFC, oportunizada pelo Programa de Residência em Medicina de Família e Comunidade.

A Residência em Medicina de Família e Comunidade mantém-se em processo de expansão no país e já pode ser encontrada em algumas das principais capitais do Brasil. Pode-se perceber que o Programa ofertado pela Secretaria Municipal de Saúde do Rio de Janeiro já apresenta uma sólida configuração, buscando ampliar a oferta de vagas em prol da garantia de equidade e integralidade do cuidado. Assim, este artigo contribui para a divulgação deste campo de atuação e da sua magnitude no país, refletindo em melhora do acesso à saúde de forma integral e equânime, em conformidade com os princípios do SUS. Também se espera que esforços no sentido de problematizar a formação de recursos na área de MFC sejam aprofundados, resultando em melhorias de acesso à saúde de qualidade.

\section{Referências}

1. Brasil. Ministério da Saúde. Secretaria de Políticas de Saúde. Projeto Promoção da Saúde. As Cartas da Promoção da Saúde. Brasília: Ministério da Saúde; 2002.

2. Brasil. Constituição da República Federativa do Brasil [Acesso 2016 Abr 22]. Disponível em https://www.imprensaoficial.com.br/downloads/ pdf/Constituicoes_declaracao.pdf

3. Brasil. Lei 8.080, de 19 de setembro de 1990 [Acesso 2016 Jun 18]. Disponível em: http://www.planalto.gov.br/ccivil_03/leis/L8080.htm

4. Nunes A, Santos JRS, Barata RB, Vianna SM. Medindo as desigualdades em saúde no Brasil: uma proposta de monitoramento. Brasília: OPAS, IPEA; 2001. 
5. Franco TB, Merhy EE. Programa Saúde da Família (PSF): contradições de um programa destinado à mudança do modelo tecnoassistencial. In: Merhy EE, Magalhães Júnior HM, Rimoli J, Franco TB, Bueno WS, orgs. O trabalho em saúde: olhando e experienciando o SUS no cotidiano. São Paulo: Hucitec; 2003.

6. Janudis MA, Blasco PG, Haq C, Freeman J. Formando médicos para a Medicina de Família e Comunidade. Rev Bioét. 2007;15(1):27-36.

7. Brasil. Ministério da Saúde. Secretaria de Assistência à Saúde. Coordenação de Saúde da Comunidade. Saúde da Família: uma estratégia para a reorientação do modelo assistencial. Brasília. Ministério da Saúde; 1997. 36 p.

8. Starfield B. Primary Care: Concept, Evaluation and Policy. New York: Oxford University Press; 1992.

9. Mello GA, Mattos ATR, Souto BGA, Fontanella BJB, Demarzo MMP. Médico de família: ser ou não ser? Dilemas envolvidos na escolha desta carreira. Rev Bras Educ Med. 2009;33(3):475-82. http://dx.doi.org/10.1590/S0100-55022009000300017

10. Campos CEA. Os Princípios da Medicina de Família e Comunidade. Rev APS. 2005;8(2):181-90.

11. Conil EM. Ensaio histórico-conceitual sobre a Atenção Primária à Saúde: desafios para a organização de serviços básicos e da Estratégia Saúde da Família em centros urbanos no Brasil. Cad Saúde Pública. 2008;24 Suppl 1:S7-S16.

12. Tanaka OY, Oliveira VE. Reforma(s) e Estruturação do Sistema de Saúde Britânico: lições para o SUS. Saúde Soc. 2007;16(1):7-17.

13. Gusso GDF, Bensenor IJM, Olmos RD. Terminologia da atenção primária à saúde. Rev Bras Educ Med. 2012;36(1)93-9.

14. Anderson MIP, Gusso G, Castro Filho ED. Medicina de Família e Comunidade: especialistas em integralidade. Rev APS. 2005;8(1):61-7.

15. Rodrigues RD, Aguilera CE, Anderson MIP. Formação e qualificação do Médico de Família e Comunidade através de Programas de Residência Médica no Brasil, hoje: Considerações, Princípios e Estratégias. Rio de Janeiro: Sociedade Brasileira de Medicina de Família e Comunidade; 2005.

16. Sociedade Brasileira de Medicina de Família e Comunidade. A Medicina de Família e Comunidade: O Que, Como, Quando, Onde, Por que. Rio de Janeiro: Sociedade Brasileira de Medicina de Família e Comunidade; 2004 [acesso 2016 Jul 29]. Disponível em: http://www. sbmfc.org.br/media/file/documentos/medicina_de_familia.pdf

17. Arias-Castillo L, Toro CB, Freifer S, Fernández MA. Perfil do médico de família e comunidade: definição ibero americana. Santiago de Cali, Colômbia:Wonca; 2000.

18. Anderson MIP, Demarzo MMP, Rodrigues RD. A Medicina de Família e Comunidade, a Atenção Primária à Saúde e o Ensino de Graduação: recomendações e potencialidades. Rev Bras Med Fam Comunidade. 2007;3(11):157-72. http://dx.doi.org/10.5712/rbmfc3(11)334

19. Soranz DR. Programa de Residência em Medicina de Família e Comunidade do município do Rio de Janeiro. Rev Bras Med Fam Comunidade. 2015;30(9):67-71 [Acesso 2016 Mar 2]. Disponível em: http://www.rbmfc.org.br/rbmfc/article/view/882

20. Falk JW. A medicina de família e comunidade e sua entidade nacional: histórico e perspectivas. Rev Bras Med Fam Comunidade. 2004;1(1):5-10 [Acesso 2015 Maio 21]. Disponível em: https://rbmfc.org.br/rbmfc/article/view/2

21. Brasil. Ministério da Saúde. Portaria oㅜ 1.143/, de 7 de julho de 2005. Apoia programas de residência médica em Medicina de Família e Comunidade (PRM-MFC), por meio do Programa de Bolsas para a Educação pelo Trabalho, do Ministério da Saúde. Brasília: Ministério da Saúde; [Acesso 2016 Jul 28]. Disponível em: http://bvsms.saude.gov.br/bvs/saudelegis/gm/2005/prt1143_07_07_2005.html

22. González Blasco P, Roncoletta AFT, Moreto G, de Benedetto MAC, Levites M, Janaudis MA. SOBRAMFA has promoted family medicine education in Brazil since 1992. Med Educ. 2008;42(1):115. http://dx.doi.org/10.1111/j.1365-2923.2007.02929.x

23. Fernandes CLC, Lopes JMC, Curra LCD, Mattos LFC. Oficina para Capacitar Preceptores em Medicina de Família e Comunidade: uma estratégia para qualificar a formação em MFC e a assistência em APS. Rev Bras Med Fam Comunidade. 2007;3(11):173-9. http://dx.doi. org/10.5712/rbmfc3(11)335

24.SBMFC. Histórico da Medicina de Família e Comunidade [Acesso 2016 Jul 31]. Disponível em: http://www.sbmfc.org.br/default.asp?site_ Acao $=$ mostraPagina\&paginald $=3$

25. SBMFC. Sociedade Brasileira de Medicina de Família e Comunidade [Acesso 2016 Jul 27]. Disponível em: http://www.sbmfc.org.br/

26. Brasil. Decreto № 80.281, de 5 de Setembro de 1977. Brasília: Presidência da República; 1977 [Acesso 2016 Jul 22]. Disponível em: http://www.planalto.gov.br/ccivil_03/decreto/1970-1979/D80281.htm

27. Brasil. Lei oㅜ 12.871, de 22 de outubro de 2013. Programa Mais Médicos [Acesso 2016 Jul 13]. Disponível em: www.planalto.gov.br/ ccivil_03/_ato2011-2014/2013/Lei/L12871.htm 
28. Brasil. Ministério da Educação. Conselho Nacional de Educação. Câmara de Educação Superior. Resolução CNE/CES n4 de 7 de novembro de 2001. Institui diretrizes curriculares nacionais do curso de graduação em Medicina. Brasília: Ministério da Educação; 2001.

29. Prefeitura do Rio de Janeiro. Medicina de Família dará bônus de 10\% para ingresso em outras áreas Rio de Janeiro: Residência de Medicina de Família e Comunidade do Município do Rio de Janeiro; 2015 [Acesso 2016 Jul 27]. Disponível em: http://www.rmfcrio. org/2015/03/medicina-de-familia-dara-bonus-de-10.html

30. Brasil. Ministério da Saúde. Editais de Residência de Medicina de Família e Comunidade. Brasília: Ministério da Saúde; 2016 [Acesso 2016 Mar 2016]. Disponível em: http://portalsaude.saude.gov.br

31. Simas KBF, Simões PP, Gomes AP, Costa AAZ, Pereira CG, Siqueira-Batista R. (Bio)ética e Atenção Primária à Saúde: estudo preliminar nas Clínicas da Família no município do Rio de Janeiro, Brasil. Ciênc Saúde Coletiva. 2016;21(5):1481-90. http://dx.doi.org/10.1590/141381232015215.00332015

32. Poli Neto P.O Programa de Residência em Medicina de Família e Comunidade do município de Curitiba. Rev Bras Med Fam Comunidade. 2014;9(31):192-4. http://dx.doi.org/10.5712/rbmfc9(31)904

33. Lermen Júnior N. O Programa de Residência em Medicina de Família e Comunidade do município de Florianópolis. Rev Bras Med Fam Comunidade. 2014;9(32):300-3. http://dx.doi.org/10.5712/rbmfc9(32)992

34. Castro VS, Nóbrega-Therrien SM. Residência de Medicina de Família e Comunidade: uma estratégia de qualificação. Rev Bras Educ Med. 2009;33(2):211-20. http://dx.doi.org/10.1590/S0100-55022009000200008

35. Prado Júnior JC. Desafios para a expansão de programas de residência em Medicina de Família e Comunidade: a experiência carioca. Rev Bras Med Fam Comunidade. 2015;10(34):1-9. http://dx.doi.org/10.5712/rbmfc10(34)1105

36. Andrade ALA, Oliver LL, Melo TP. Implantação do Programa de Residência em Medicina de Família e Comunidade da Secretaria Municipal de Saúde do Rio de Janeiro. Ciênc Saúde Coletiva. 2016;21(5):1471-80. 